
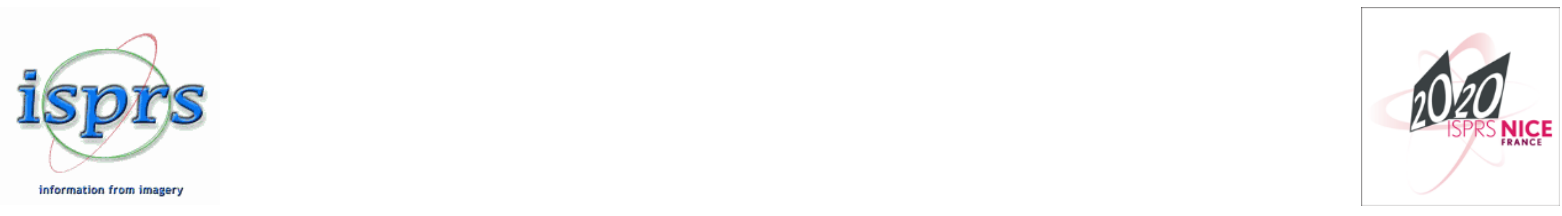

\title{
Preface
}

\section{Technical Commission IV on Spatial Information Science}

\author{
S. Zlatanova ${ }^{1 *}$, S. Dragicevic ${ }^{2}$, G. Sithole ${ }^{3}$ \\ ${ }^{1}$ President, University of New South Wales, Sidney, Australia - \\ s.zlatanova@unsw.edu.au \\ 2 Vice President, Simon Fraser University, Burnaby, Canada - suzanad@sfu.ca \\ ${ }^{3}$ Secretary, GeoVariant, Cape Town, South Africa - georgesithole@geovariant.com
}

The unusual circumstances created by the coronavirus pandemic has impacted recent activities of Commission IV. The situation also provides an excellent opportunity to connect the work of the Commission to addressing an important global problem. Managing the social and economic challenges brought by increased complexity and interconnectivity of activities in human society requires new dimensions of analysing information and specifically spatial information. The increased pressure on the usage of geographic space, maintaining sustainable development and creating liveable community environments increases the requirements for spatial decision-making tools. Commission IV Spatial Information Science (2016-2020) is dedicated to advance research activities in spatial information sciences for modelling, structuring, management, analysis, visualization and simulation of (big) data with focus on the third spatial dimension and taking into consideration dynamic changes. Special attention is given to linking information about real-world physical phenomena with societal, organizational and legal information in order to address the complexity of issues in their entirety. The Commission has contributed to advancements in data modelling, data fusion and management, visualization (web-based, VR and AR), simulation and city analytics, and $3 \mathrm{D}$ applications. The work had largely been implemented in cooperation with international organizations such as FIG, UDMS, 3DGeoinfo, ICA, OGC, ISO and Web3D.

The Commission consists of 10 scientific areas of research that is coordinated by 10 working groups (WG) as follows - WG1: Strengthen the work on multidimensional spatial model and representations towards seamless data fusion; WG2: Advance the semantic modelling, development and linking of ontologies; WG3: Intensify research into data interpretation, quality and uncertainty modelling; WG4: Strengthen research

\footnotetext{
${ }^{*}$ Corresponding author
} 
on crowdsourced data and public participation, towards community-driven and participatory applications, collaborative mapping and use/usability of maps; WG5: Strengthen research on seamless indoor/outdoor location-based services, navigation and tracking, and analysis of human movement; WG6: Advance interoperable Internet of Things, Sensor web, SDI and linked data; WG7: Advance research on spatial data types, indexing methods and analysis to further contribute to development of spatial DBMS for management and analysis of multi-dimensional data; WG8: Encourage the use of functional programming and streaming algorithms in development of demos and applications as well as parallel and distributed processing paradigms; WG9: Advance visual analytics, online multi-dimensional visualization on mobile and desktop devices, considering human-centred applications, privacy and security issues; WG10: Advance knowledge on the use of spatial information (BIM/GIS) for urban modelling; ICWG IV/III: Global Mapping: Updating, Verification and Interoperability with the mission to promote the development of advanced methodologies and applications for the update, verification and interoperability of geospatial databases.

The papers received for the ISPRS congress reflect the above-mentioned scientific research areas. The reported research ranges from advancements in new and emerging theories, through experiments and analysis to demonstration of technologies in different applications. The research was captured through papers and abstracts published in the collection of ISPRS Annals and ISPRS Archives. The papers and abstracts were selected for inclusion through a rigorous peer-review process. The ISPRS Annals contain 29 papers and the ISPRS Archives contain 114 papers. The diversity of the research topics presented in the published papers clearly indicate the wide range of topics within the field of Spatial Information Science. A rigorous peer-review process by the ISPRS TC IV Scientific Committee Working Group Chairs ensured hight quality and scientific innovation.

\section{TC IV Scientific Committee}

Giorgio Agugiaro Delft University of Technology, Delft the Netherlands, Jamal Jokar Arsanjani Aalborg University, Aalborg, Denmark, Pawel Boguslawski Wroclaw University of Science and Technology, Wroclaw, Poland, Martin Breunig Karlsruhe Institute of Technology, Karlsruhe, Germany, Maria Antonia Brovelli Politecnico di Milano, Milan, Italy, Sidonie Christophe IGN-France, Paris, France, Arzu Coltekin University of Zurich, Zurich, Switzerland, Mahmoud R. Delavar University of Tehran, Tehran, Iran, Mulhim Al Doori American University, Dubai, UAE, Suzana Dragicevic Simon Fraser University, Burnaby, Canada, Eric Guilbert Université Laval, Québec, Canada, Cidalia Costa Fonte University of Coimbra, Coimbra, Portugal, James Haworth University College London, London, United Kingdom, Umit Isikdag Mimar Sinan Fine Arts University Istanbul, Istanbul, Turkey, Ivana Ivanova, Curtin University, Perth, Australia, Zhizhong Kang China University of Geosciences, Beijing, China, Kourosh Khoshelham University of Melbourne, 
Melbourne, Australia, Mila Koeva University of Twente, Enschede, The Netherlands, Margarita Kokla National Technical University of Athens, Athens, Greece, Yaolin Liu Wuhan University, Wuhan, China, Marguerite Madden University of Georgia, Athens, Georgia, USA, Mir Abolfazl Mostafavi Université Laval, Quebec, Canada, Gerhard Navratil Technical University Wien, Vienna, Austria, Dev Raj Paudyal University of Southern Queensland, Toowoomba, Australia, Christopher Pettit University of New South Wales, Sydney, Australia, Antonia Spano, Politecnico di Torino, Italy, George Sithole GeoVariant, Cape Town, South Africa, Emmanuel Stefanakis University of Calgary, Calgary, Canada, Wei Tu, Shenzhen University, Shenzhen, China, Giuseppina Vacca University of Cagliari, Cagliari, Italy, Lucia Diaz Vilarino University of Vigo, Vigo, Spain, Sarah Wise, University College London, London UK, Hao Wu National Geomatics Center of China, Beijing, China, Sisi Zlatanova University of New South Wales, Sydney, Australia, Xiao Guang Zhou Central South University, Changsha, Hunan China 